\title{
Retorica dell'artefatto concreto. Per un design di prodotto incardinato su uso, identità tangibile, stratificazione di senso
}

\section{Sintesi}

Nella nostra quotidianità è enormemente cresciuto l'impatto delle tecnologie presenti nei prodotti materiali e distribuite all'interno di prodotti/sistemi/servizi immateriali. Oggetti legati a tecniche semplici o complesse esistono da sempre, ma da circa tre decadi a questa parte si assiste da una parte alla pervasività dei prodotti ad alto contenuto tecnologico, ostentativi e iperprestazionali, dall'altra alla apparente riduzione del sostrato fisico a favore di un'aumentata componente immateriale e virtuale. Tuttavia la nostra contemporaneità, pur nel dilagante rimando al virtuale, non ha rinunciato per nulla alla fisicità di cose e prodotti. All'interno di un assetto disciplinare del design esteso e frammentato, ci si propone l'obiettivo di riannodare il filo di un possibile discorso intorno alla concretezza tangibile degli oggetti, indirizzandolo in particolare alla costruzione di pedagogie rivolte agli studenti progettisti. Da qui la selezione in particolare dei temi dell'uso, dell'identità tangibile dei prodotti, della sostanza di cui sono fatti, intesa nel duplice senso di sostrato materico e di stratificazione di senso.

Parole chiave: design del prodotto, pedagogia del design, cultura materiale

Rhetoric of concrete artifacts.

For a product design hinged on use, tangible identity, meaning stratification

\section{Abstract}

The immaterial impact of technologies on people daily experience has enourmously increased over time. At the same time people are still immersed in the tangible properties of matter shaping products and product systems. The last three decades have witnessed the novelty of an all-pervading stream of conspicuously technological products, whose material substratum seems progressively reduced in favour of increasing immaterial and virtual performances. However, the claims of a concurrent design culture to be built on the all-pervading preminence of immateriality are rather paradoxically faced by the dominance of matter. To guide design novices to fully understand the opportunities of matter - as well as to cope with the counterpart of immateriality - is therefore assumed that education in design more than ever advocates an integrated approach developed on the terrain of the tangibility of objects and of the human dimension of the relation with man made things. Hence the selection of the themes of use, of the tangible identity of products, of their substance meant as material substratum and stratification of meaning.

Keywords: Social design, Social Work, social intervention, young people at risk of exclusion.

\footnotetext{
Professore ordinario di Disegno industriale al Politecnico di Milano, Scuola del Design. Afferisce al Dipartimento di Design, dove è membro della Sezione Design e culture. Laureata in Architettura con Lode, è Dottore di ricerca in Disegno industriale. Per il quadriennio 2012-15 è stata Presidente del Corso di Laurea in Design del Prodotto Industriale e Corso di Laurea Magistrale in Design del Prodotto per l'Innovazione. Tra i suoi principali interessi di studio i fondamenti del disegno industriale, i metodi di ricerca per il design e le metodologie di didattica del progetto. Email: silvia.pizzocaro@polimi.it
} 


\section{Introduzione}

L'assunto che l'impatto delle tecnologie (informatica, elettronica, robotica, telecomunicazioni, bioingegnerie, tecnologie dei materiali avanzati) produrrebbe un sempre più avanzato assottigliarsi della materialità del mondo ha comportato un quadro di riferimento per il design di prodotto proiettato oltre l'"esibizionismo funzionalista" (Bassi, 2010), favorendo un'enfasi crescente, finanche esclusiva, sulle caratteristiche della performance dei prodotti e sulle componenti comunicativo-informative. In questa cornice ha preso grande forza la visione di una contrazione dell'universo degli oggetti fisici, sostituiti da processi e servizi immateriali.

Senza entrare nel merito di un'interpretazione critica, qui ci limitiamo a considerare che la maggior parte degli oggetti - artefatti, utensili, attrezzi, ausili - di fatto presenta da sempre un sostrato riferibile a un qualche tipo di tecnologia. Ciò che oggi è cambiato è il livello di pervasività della tecnologia, e con questo la pretesa che il fenomeno si attui nella dimensione dell'immaterialità.

La quotidianità individuale e collettiva rimane tuttavia estensivamente pervasa di consistenza materiale da considerare non contro bensì più semplicemente insieme all'immaterialità più sopra evocata. Quella che si propone è quindi una ricognizione all'insegna della concretezza (Pizzocaro, 2016) - certamente senza pretesa di esaustività - attorno ad alcuni specifici nuclei argomentativi: $i$ temi dell'uso e dell'identità tangibile dei prodotti, intrecciati ad una riflessione sulla sostanza di cui sono fatti, intesa nel duplice senso di sostrato materico e di stratificazione di senso.

\section{Lo sfondo del discorso}

Qualche ulteriore considerazione meglio focalizzata, per quanto sempre di ordine generale, ci serve per tratteggiare lo sfondo del discorso.

Nella condizione attuale lo status dei prodotti e il loro valore non hanno più una relazione lineare con le regole dei sistemi di produzione e con i relativi costi, ma piuttosto con il potere dei segni e dei simboli veicolati sia da icone mediali che da artefatti propriamente fisici: "[...] a questo punto diventa necessaria una nuova e allargata teoria del valore: il valore di questi beni - materiali o immateriali - non può più essere determinato in base alle regole dei costi di produzione dell'economia politica tradizionale, ma piuttosto in base ad altre regole che hanno a che vedere con la valutazione della potenza dei segni [...]" (Carmagnola, 2001, p. 38). Da qui un'estetica che corrisponde "all'economia impura e finzionale del simbolico, che non riconosce più la sua base nella coppia classica valore d'uso/valore di scambio" (Carmagnola, 2001, p. 52).

Questi inediti campi di azione della progettazione (legati alle dimensioni emozionali, virtuali o multi e iperfunzionali dei prodotti) hanno innescato quella che è parsa "una crisi ontologica del design del prodotto" (Cristallo, 2015, p. 28), connessa ad un destino debole e rinunciatario della cultura stessa del design, che si muove "in spazi liminari e laterali rispetto alle grandi questioni (e ai poteri economici) del nostro tempo" (Bassi, 2010).

L'ipotesi di un destino debole della cultura progettuale si è comunque andata delineando a fronte di una contemporaneità che, pur nel dilagante rimando al virtuale, non ha rinunciato per nulla alla fisicità di cose e prodotti. In questa prospettiva, il design come disciplina e professione continua a mantenere un ruolo 
cruciale per dare forma e sostanza agli obiettivi che muovono o muoveranno le persone e le economie: un ruolo chiamato ad affrontare sul terreno del progetto le problematiche della sommatoria delle funzioni; della convergenza tecnologico-funzionale in artefatti singolari; della permanenza dell'oggetto mono-funzione; dell'avanzamento del fronte di sperimentazione delle componenti esperienziali dei prodotti (che si integrano e innestano su quelle fisiche e materiali); del consolidamento di prassi progettuali specificamente intese a cogliere e interpretare i bisogni e i desideri delle persone e a modellare i prodotti di conseguenza.

\section{Una concretezza da ri-esplorare}

Certo, in questo orizzonte la strumentalità dell'oggetto rivendicata dal funzionalismo in senso tradizionale ha perso terreno: il valore simbolico dell'oggetto ha carattere funzionale nel quadro sociale, e questo valore ha caratteri che non derivano più direttamente dal momento di produzione ma da quello del suo uso e consumo: più che di oggetti come tali si discute quindi di oggetti nei loro contesti di uso e di valorizzazione, inclusa quella che con Marrone (2002, p. 15) possiamo definire la valorizzazione estetica.

Per il progetto di prodotti, quindi, non è più questione di concepire funzioni pratiche che si conciliano con le forme: il suo compito, come osserva Marrone (2002), si estende ora alla previsione e alla prescrizione "di un consumo piuttosto che un altro, di una valorizzazione piuttosto che un'altra, di un'attribuzione di senso piuttosto che un'altra" (p. 15).

Il rimando ad uno scritto di De Fusco (2008) ci consente inoltre di riprendere - qui solo emblematicamente la questione dell'immaterialità dalla prospettiva dei servizi, beni immateriali erogati come neomerci dall'alto contenuto informativo. Su un fronte critico, De Fusco (2008) osserva che i servizi spostano il design fuori dalla cultura materiale, "occultando la specificità della disciplina con operazioni metaoperative" (p. 68) estranee alla cultura del design. Questa presa di posizione consente di osservare polemicamente che "il rimandare il design ai servizi ricorda quelle promesse politiche che spostano costantemente ad un livello superiore quanto non si vuole risolvere a livello reale" (De Fusco, 2008, p. 68).

Nella riflessione che qui proponiamo al lettore è sottesa, invece, la scelta di affrontare proprio quei livelli del reale che appaiono irrisolti, comportando l'esigenza di ri-osservare gli effetti che le tecnologie per un verso e le molteplici economie per l'altro hanno prodotto e continuano a produrre in modo estensivo sull'agenda concreta del design (Carmagnola, 2001, p. 28), proprio a partire dalle istanze della supposta avanzata della dematerializzazione.

Ci si riferisce al divorzio conclamato tra forma e funzione, all'eclissi della riconoscibilità dei prodotti, alla generazione di nuove identità, alla rideclinazione dei rapporti tra involucro e interno dell'artefatto, al passaggio dall'oggetto tecnico all'artefatto tecnologico, nell'accresciuta libertà di manovra con cui svincolare la parte funzionale di un oggetto dalla configurazione formale.

\section{Forme dell'uso e design involontario}

Guardando in particolare i fondamenti del progetto di prodotto, ci si ri-propone di orientare soprattutto gli studenti principianti al passaggio progressivo dall'intendere in modo naif il disegno industriale come "progettazione attraverso il disegno" (Dorfles, 1972, p. 122) a riconoscergli - certo in modo adeguato ai tempi - il compito di integrare quei fattori relativi all'uso, alla fruizione e alla stratificazione di senso individuale e sociale che partecipano al processo costitutivo del prodotto stesso (Maldonado, 1976, p. 9). 


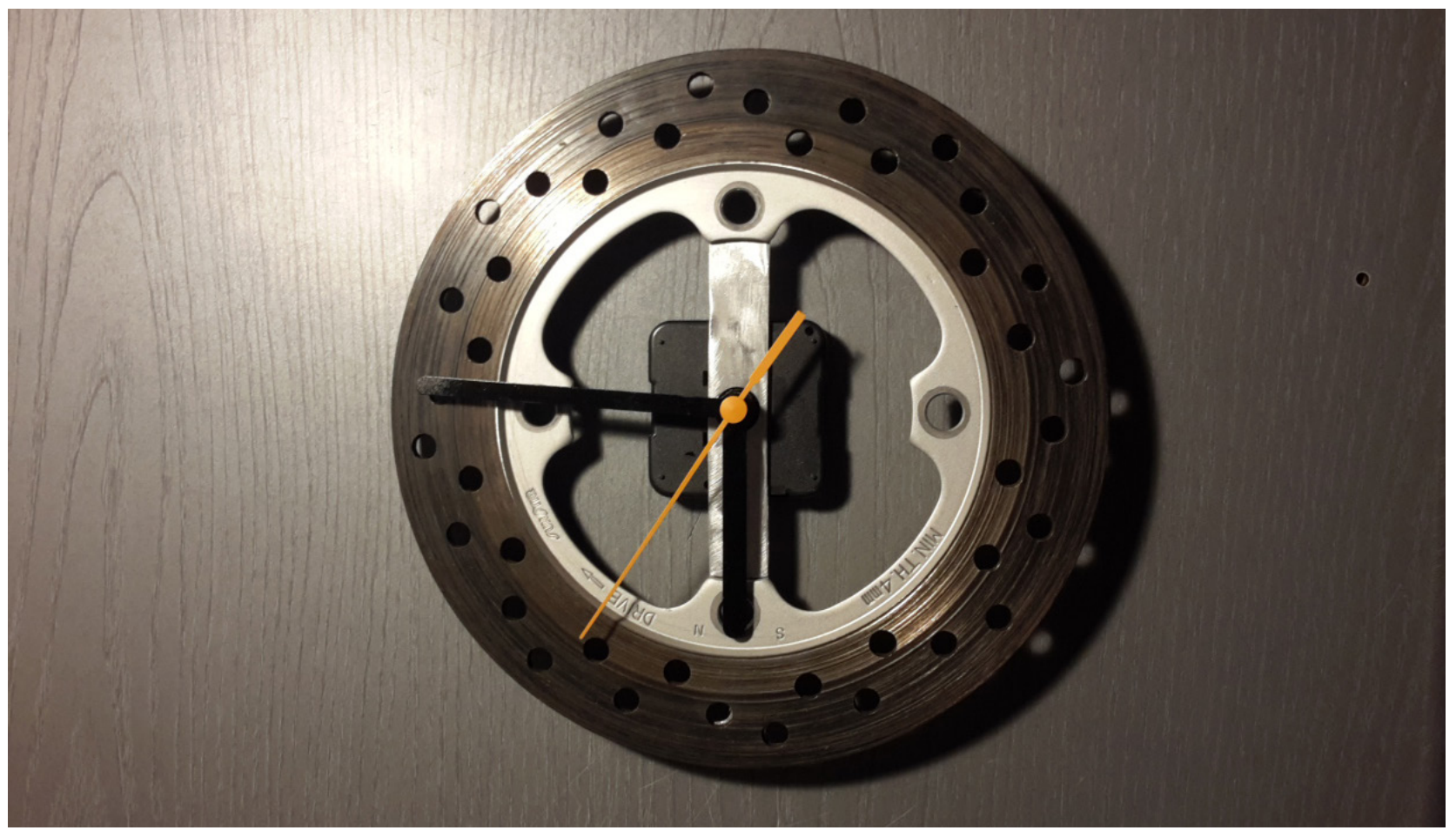

Fig. 1. Freno a disco come orologio. Esercitazione, ready-made e foto di Jacopo Fusi. Anno accademico 2015/16, Scuola del Design del Politecnico di Milano, Laboratorio di Fondamenti del progetto.

Specificamente scegliamo di prendere le mosse da una definizione parziale e indiretta di uso: "Lo scopo del design è produrre un oggetto o un sistema che sia usato da un utente" (Erlhoff \& Marshall, 2008, p. 429). I progettisti creano oggetti usati dalle persone; naturalmente questo non implica necessariamente che l'uso progettato costituirà davvero un vincolo per chi userà quegli oggetti.

Emergenti prospettive di analisi - come gli studi sugli utenti (Pizzocaro, 2015) - rimandano estesamente l'uso che gli utenti effettivamente fanno, o non fanno, dei prodotti progettati per loro, inclusi gli usi imprevisiti o indesiderati. Gli utenti possono infatti interpretare l'uso attraverso forme di "design non intenzionale" (Brandes \& Erlhoff, 2006), con cui ci si riferisce alla pratica abituale di decodificare creativamente l'uso degli oggetti, contribuendo a personali, inedite, spesso improbabili ma anche assai efficaci ri-destinazioni d'uso (fig. 2, 3, 4).

II design involontario disvela un sorta di plurifunzionalità potenziale per oggetti apparentemente privi di ambiguità, generando soluzioni frutto dell'inventività (da parte delle persone) e dello slittamento funzionale (rispetto all'originaria vocazione). Questa pratica esprime pertanto la necessità di ricorrere a usi modellati su esigenze ad hoc, riconoscendo alle persone il potere di sovvertire creativamente l'uso degli oggetti d'uso quotidiano, senza necessariamente operare sul piano della riconfigurazione morfologica.

\section{L'uso come manipolazione, gesto tecnico e appropriazione}

Adottando una prospettiva antropo-centrica, ci si chiede come le persone intendono l'uso di un prodotto, quale significato vi riconoscono, indipendentemente dalle prescrizioni d'uso previste dai progettisti. Questa domanda ci sembratuttora comportare una riflessione sull'uso come gesto elementare e prolungamento del gestire tecnico legato alla prensione. 


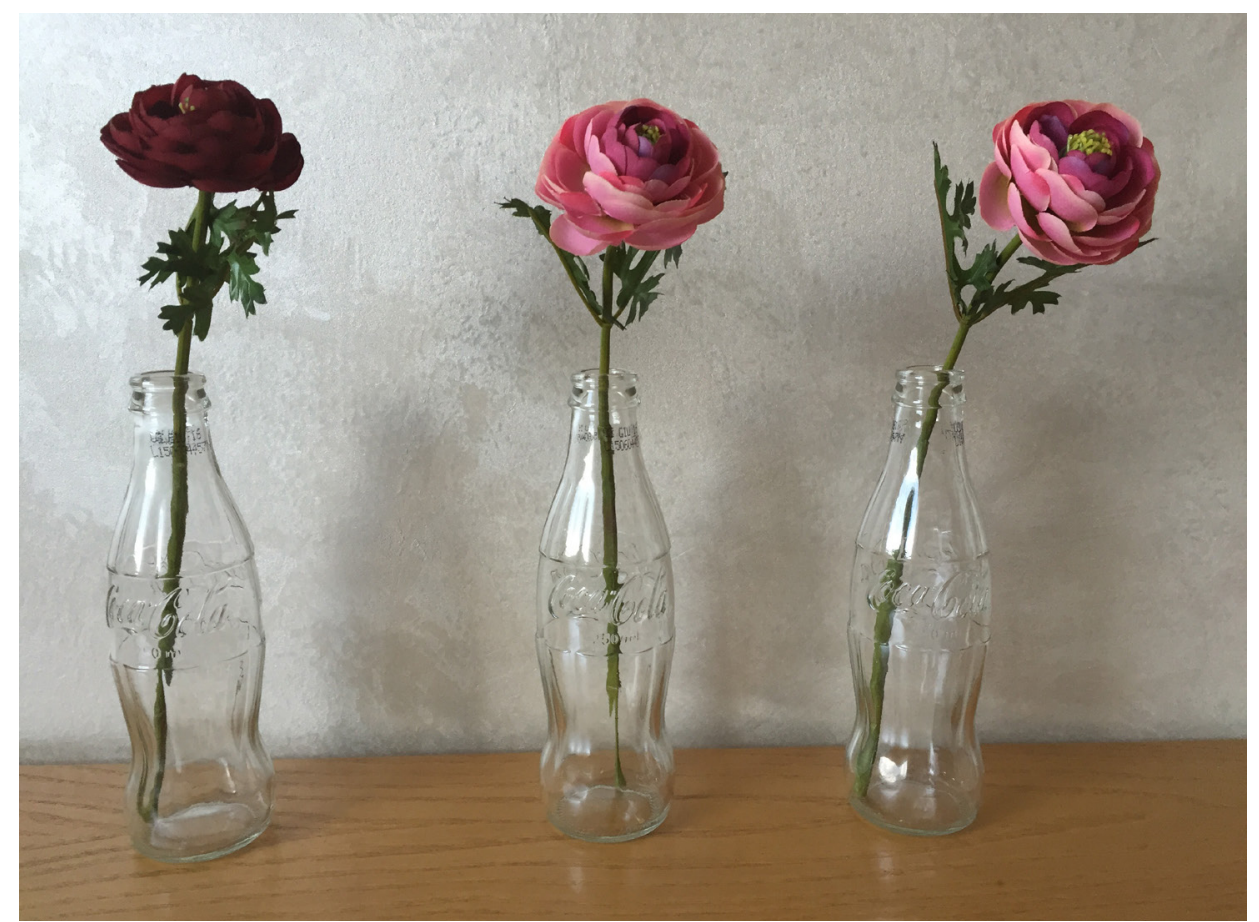

Fig. 2. Bottiglia-vaso. Esercitazione e foto di Margherita Stanga. Anno accademico 2015/16, Scuola del Design del Politecnico di Milano, Laboratorio di Fondamenti del progetto.

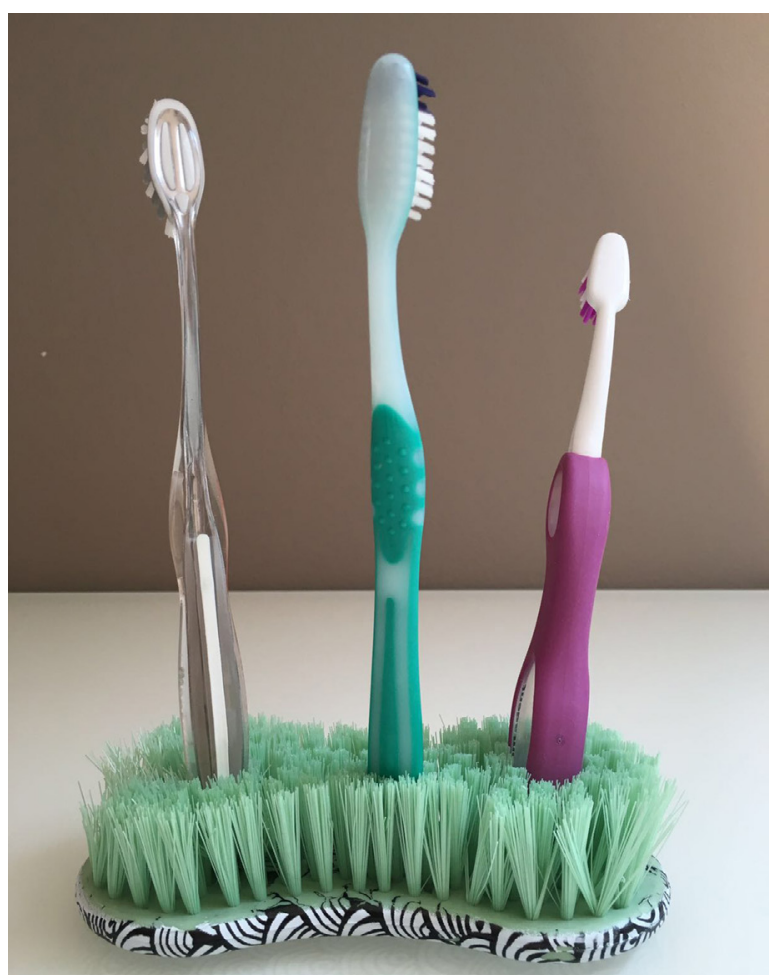

Fig 3 Spazzola a setole rigide con funzione di portaspazzolini. Esercitazione e foto di Elisabetta Carrara. Anno accademico 2015/16, Scuola del Design del Politecnico di Milano, Laboratorio di Fondamenti del progetto.

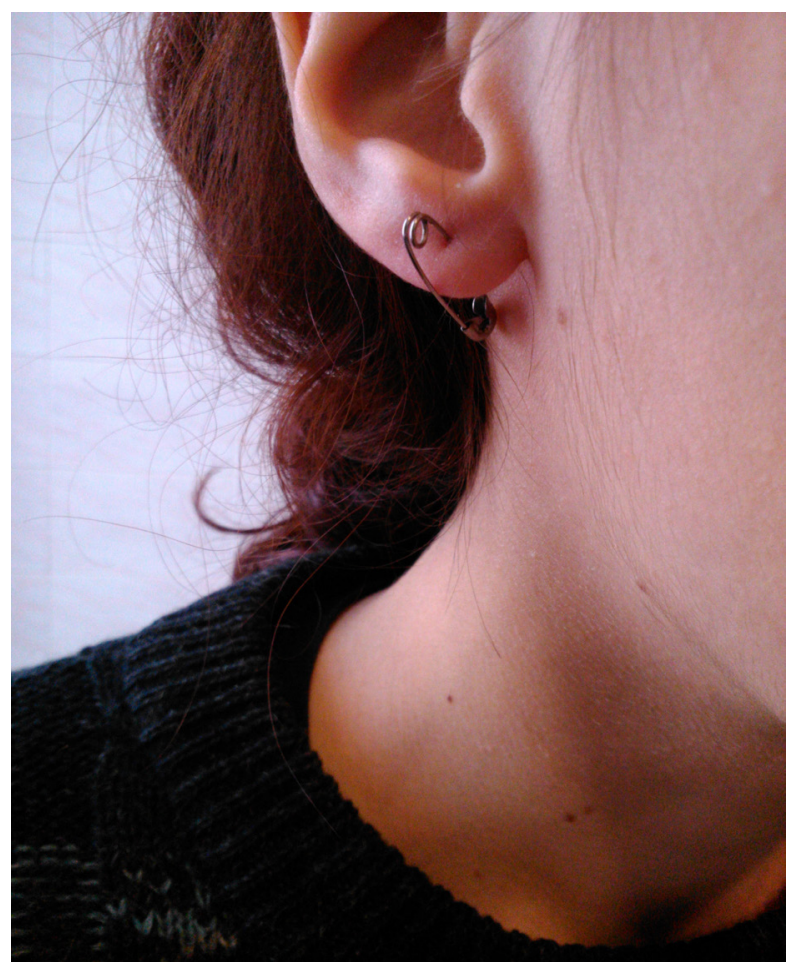

Fig 4. Spilla da balia usata come orecchino.

Esercitazione e foto di Elena Bastia. Anno accademico 2015/16, Scuola del Design del Politecnico di Milano, Laboratorio di Fondamenti del progetto 
Sul tema del gesto, Leroi-Gourhan (1977) ha osservato che l'utensile esiste realmente "solo nel gesto che lo rende tecnicamente efficace" (p. 278). La priorità del gesto prensile è inoltre sufficiente a isolare i Primati dal resto dei mammiferi e a dare avvio al comportamento operazionale dell'uomo. II confine tra Primati e uomo sta proprio nell'apparizione dell'utensile che - come osservava Leroi-Gourhan (1977) - "è in qualche modo trasudato" (p. 283), quando nel corso dell'evoluzione la mano in motilità diretta cessa di essere utensile $e$ l'utensile manuale, ora esteriorizzato, si separa dal gesto motore.

In una nostra lettura sommaria, adattata alla misura della presente riflessione, qui si assume che gli oggetti trovano nella mano, e in particolare nella prensione, il tramite con cui dare corpo all'uso.

II movimento manuale, che immaginiamo concreto, fisico, con la forza richiesta da pressione, stretta, percussione, presa, pervade sia il mondo dei semplici oggetti d'uso che quello dei dispositivi tecnologici con cui esercitare comandi e controlli, produrre input immateriali, generare funzioni informative e virtuali: come testimoniano lo spostamento del mouse sospinto sul piano della scrivania, la pressione leggera impressa sulle tastiere, lo schiacciamento deciso e volitivo sugli interruttori, lo sfioramento dei comandi sulle superfici prive di tasti, la presa imposta dagli strumenti per la scrittura, la precisione dei movimenti fini richiesti dai dispositivi miniaturizzati, lo sfioramento con i quali la mano si avvicina e realizza il contatto sulle interfacce prive di appigli per la prensilità.

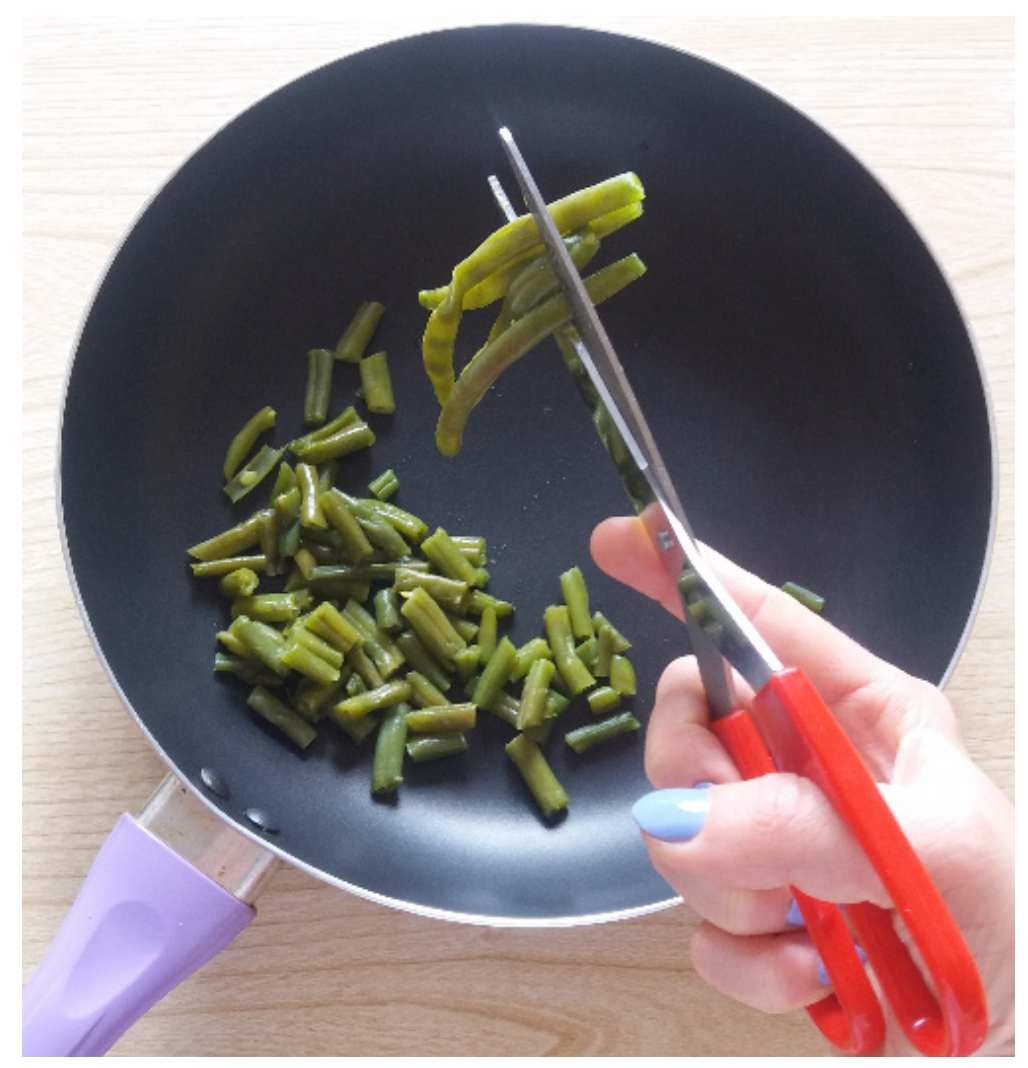

Fig. 5. Forbice impugnata come utensile per cucina.Esercitazione e foto di Sofia Costa. Anno accademico 2015/16, Scuola del Design del Politecnico di Milano, Laboratorio di Fondamenti del progetto. 
La natura del semplice gesto con cui afferriamo gli oggetti non sembra subire drastiche trasformazioni in relazione alla complessità funzionale eventualmente assunta dagli artefatti tecnologici, salvo certamente implicare minore o del tutto trascurabile fatica muscolare. Quanto sintetizzato da Maldonado (1992) appare attualizzabile: "Non c'è dunque scampo al vincolo della fisicità. [...]. È irragionevole congetturare [...] che gli uomini, nel loro vivere di ogni giorno, potrebbero sbarazzarsi definitivamente della elementare e persino, si dice, troppo rozza e ingenua esigenza di voler sempre e comunque toccare con mano le cose di questo mondo" (p. 12).

Con l'eccezione intuibile del passaggio ai comandi vocali, destinati a introdurre una trasformazione radicale nella relazione con gli oggetti, l'uso ci appare quindi in misura preponderante tuttora legato al movimento delle mani, al tocco delle dita, a precise sequenze di gesti. La pressione e prensione della mano e delle dita racconta un'esperienza dell'uso che è stata, e in larga misura continua a rimanere, manipolazione.

\section{Re-visioni dell'uso}

Sulla scorta delle prospettive aperte sul progetto dell'uso, ma anche delle inedite centralità dell'uso oltre l'oggetto, dell'acquisizione di design dell'esperienza, degli sviluppi dell'affordance come insieme delle proprietà di un artefatto che ne determinano le possibilità d'uso, si delineano nuove formulazioni che non dipendono né dai modelli mentali dei progettisti né dagli schemi di comportamento delle persone e che porterebbero a definizioni di "uso attraverso l'uso" (Redström, 2008, p. 410).

I progettisti non progettano solo la forma delle cose, ma anche la forma dell'uso: è noto che, per esempio, quando si progetta una sedia come oggetto fisico contemporaneamente si progetta un particolare atto del sedersi.

La differenza non rimanda solo alla distinzione tra forma e funzione, che si riferiscono rispettivamente a ciò che un oggetto è (''insieme integrato di una seduta, delle gambe e dello schienale) o fa (consentire la seduta). Quello che meglio si specifica è il concetto di azione: l'azione del sedersi non è un attributo della sedia, è l'atto che potremmo elettivamente compiere con quell'oggetto. È quella che ci sembra di poter chiamare la vocazione che si assegna alla sedia.

La misura con cui un utente può aderire a questa vocazione è una questione di possibilità: possiamo essere efficacemente invitati o indotti a sederci nel modo prescritto, oppure no. Possiamo riadattare in modo più personale la nostra idea del sedersi. Possiamo usare una sedia per altri scopi (fig. 6).

L'incontro tra le forme di uso mediato dalle persone e le potenzialità d'uso inscritte in un prodotto si gioca quindi almeno su tre livelli:

- ciò che si può fare con quell'oggetto;

- ciò che si sa fare, per convenzione, abitudine o per familiarità;

- ciò che si vuole fare (indipendentemente dall'uso prescritto dai progettisti).

Progettare un artefatto comporta quindi non solo concepire un oggetto in sé, ma inscrivervi delle vocazioni d'uso aperte. 


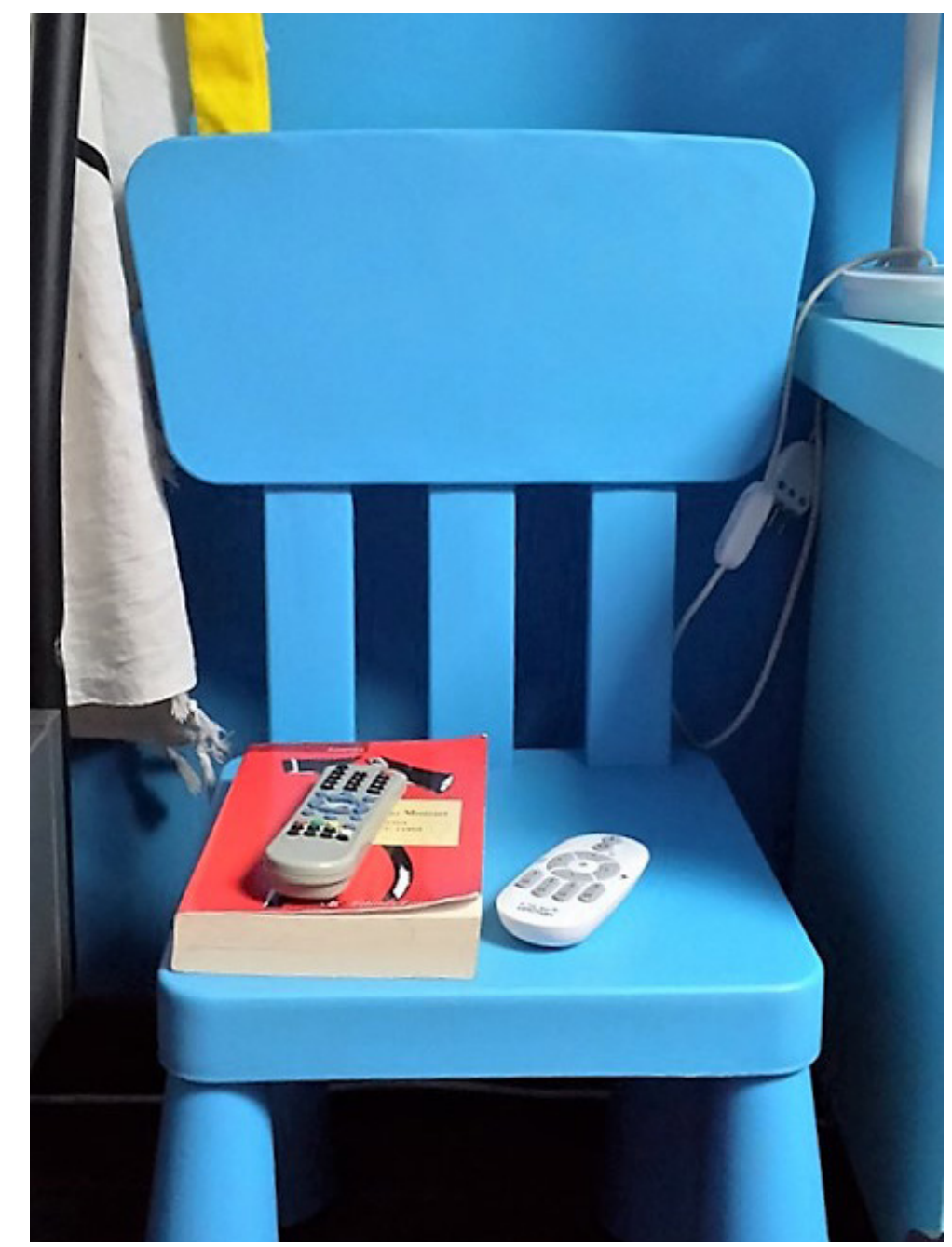

Fig. 6. Sedia per bambini riconvertita a comodino. Esercitazione e foto di Altea Troiano. Anno accademico 2015/16, Scuola del Design del Politecnico di Milano, Laboratorio di Fondamenti del progetto.

Per completezza aggiungiamo che è rubricabile anche il livello del dover fare, che può corrispondere, secondo Fontanille (2002, p. 88), all'approccio dell'ergonomia che, a partire dalla rappresentazione schematica di un gesto, iscrive un vincolo nella materia e nella forma dell'oggetto.

\section{Encomio del vissuto concreto}

Se l'orizzonte di nuove progettualità uso-centriche per un verso si sposta sul piano di un futuro riempito di vocazioni all'uso, dall'altro ci consente di operare una rivalutazione degli usi passati, con cui qui si intende sia la dimensione di routine d'uso che quella della traccia fisica lasciata sugli oggetti usurati.

La familiarità con l'uso corrisponde alla confortante frequentazione degli oggetti quotidiani, alla relazione profonda con i propri oggetti, posseduti, pienamente sfruttati nelle loro funzionalità, investiti di apprezzamento e, non di rado, di affetto. 


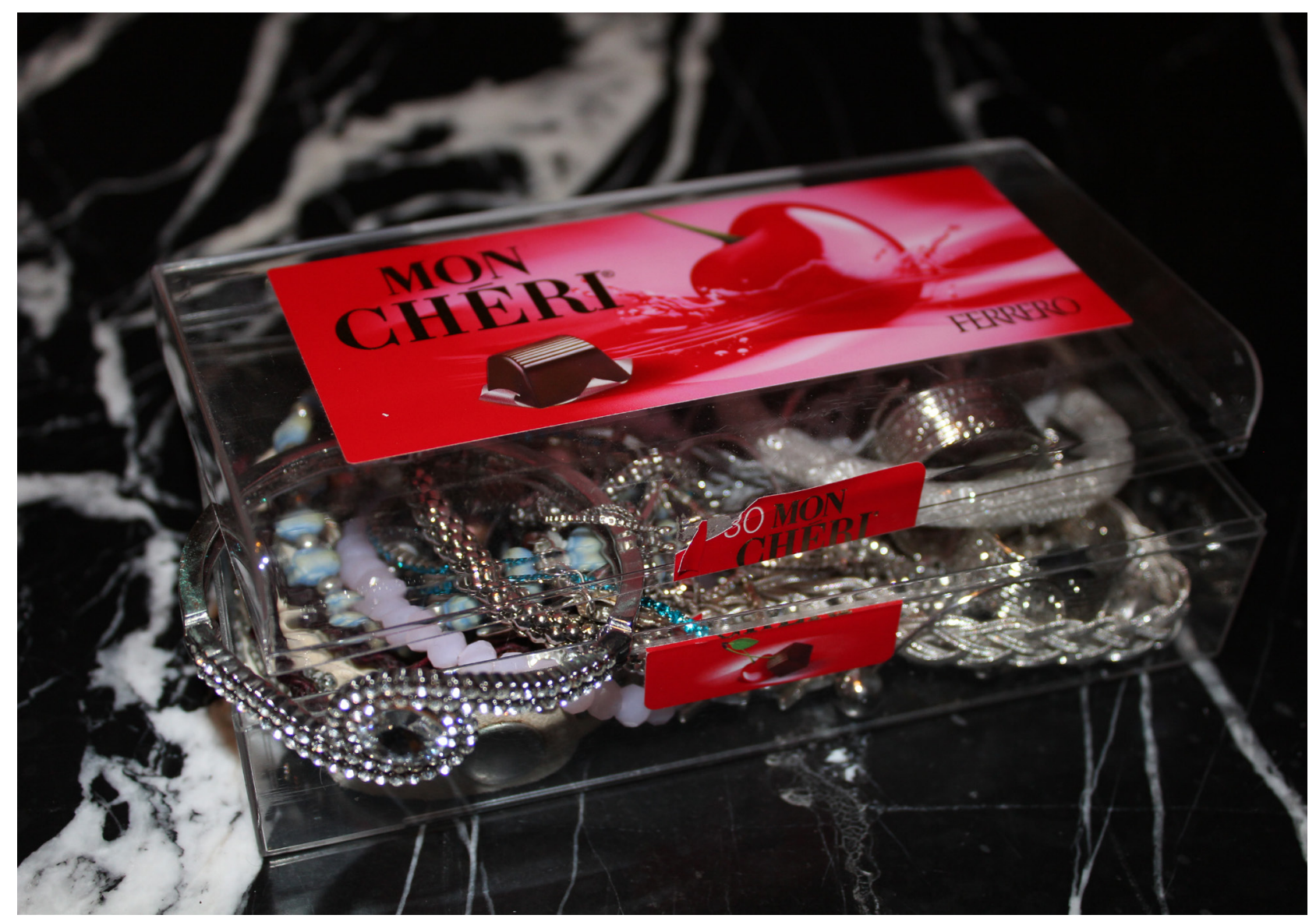

Fig. 7. Scatola per cioccolatini riciclata e usata come porta-gioielli. Esercitazione e foto di Ivana Riva. Anno accademico 2015/16, Scuola del Design del Politecnico di Milano, Laboratorio di Fondamenti del progetto.

Dal punto di vista delle proprietà formali degli oggetti, l'effetto visibile dell'uso è quello che genericamente possiamo intendere come patina, la traccia superficiale. Qui si riprende ampiamente il noto saggio di Fontanille (2002) che ricorda che "in senso proprio, la patina è lo strato formato da un composto di rame che si deposita col tempo sulla superficie degli oggetti contenenti una parte di questo metallo. In senso traslato, la parola designa qualsiasi alterazione superficiale che il tempo arreca agli oggetti costituiti da un materiale duro, stabile e in genere immutabile" (p. 88). La patina è dunque sia un'espressione del "tempo che passa", iscritto sulla superficie esterna degli oggetti, "sia espressione del tempo che dura, testimoniato dalla solidità e dalla permanenza della materia e della struttura interna degli oggetti" (p. 88).

La patina è il segno concreto del vissuto degli oggetti, manifestazione di una memoria dell'uso che si iscrive nella materia di cui gli oggetti sono fatti. Ė un consumarsi progressivo che rende gli oggetti familiari e "umanizzati" (Fontanille, 2002, p. 72): la patina testimonia infatti usi anteriori di oggetti che recano l'impronta di quanti se ne sono serviti.

In quanto superficie di iscrizione semiotica "che agisce per ritenzione e protensione" (Fontanille, 2002, p. 72), la patina modifica le proprietà sensibili degli oggetti, esteriorizzando nuove proprietà di forma, colore, testura, che realizzano un linguaggio espressivo fatto di segni, colori sbiaditi, ammaccature, pezzi mancanti, aggiustature, rammendi tecnici. 
Anche in questo caso solo la profondità esistenziale - e non meramente strumentale - del nostro rapporto d'uso con le cose può spiegare perché possa essere così difficile disfarci dei nostri oggetti invecchiati e anacronistici. Oltre ad esprimere il tempo, infatti, le cose esprimono la nostra condotta nel tempo e possono sospendere, sincopare o interrompere il dispiegarsi uniforme del tempo stesso (Connor, 2014, p. 15).

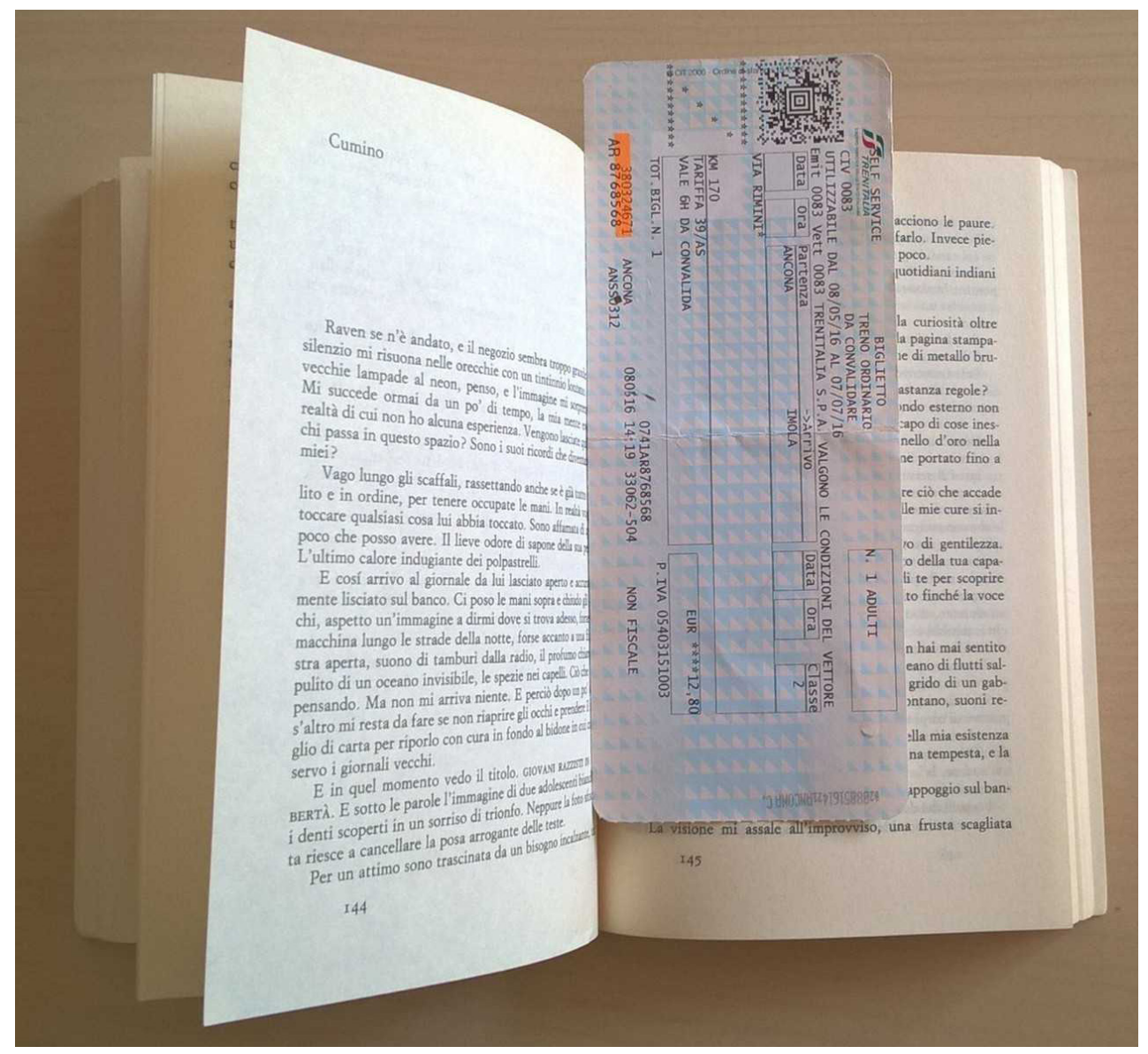

Fig. 8. Biglietto del treno riutilizzato come segnalibro. Esercitazione e foto di Francesca Veresani. Anno accademico 2015/16, Scuola del Design del Politecnico di Milano, Laboratorio di Fondamenti del progetto.

\section{Conclusioni: enfatizzare le fenomenologie di permanenza del concreto}

Superando le interpretazioni che enfatizzano acriticamente l'aspirazione verso il cambiamento e il nuovo a tutti i costi, e in parte avverso un'estetica dell'immateriale che è a volte retorica, rileviamo che è largamente trascurata una rinnovata contro-retorica profonda che rimandi a sua volta alla tanta parte dei fenomeni di permanenza e di persistenza del substrato materiale delle cose.

Per questo non ci sembra superfluo recuperare il significato e l'importanza pedagogica che può rivestire l'introduzione di una fenomenologia degli oggetti a cavallo tra impronte di usi passati e immanenti segnali di vocazione d'usi prossimi, nella cornice critica di una cultura dei prodotti che è in prima istanza realtà concreta, tangibile e significativa, anche se meno avvincente rispetto all'aspettativa generata dall'errompere e dal dispiegarsi del progetto come proiezione verso futuri virtuali. 


\section{Riconoscimenti}

Le immagini che accompagnano il testo esemplificano esercizi svolti da studenti e studentesse principianti nel Laboratorio di Fondamenti del progetto, Prof. Silvia Pizzocaro, insegnamento di Elementi di progettazione, a.a. 2015-16, presso la Scuola del Design del Politecnico di Milano. Le esercitazioni sviluppano il tema del design involontario.

\section{Bibliografia}

BASSI, A. (2010). II design dell'artefatto tecnologico. In XXI secolo, Enciclopedia Treccani on line, disponibile http://www.treccani.it/enciclopedia/il-design-dell-artefatto-tecnologico_(XXI-Secolo)/ (25.11.2018).

BRANDES, U. \& ERLHOFF, M. (2006). Non Intentional Design. Cologne, Germany: Daab Media.

CARMAGNOLA, F. (2001). Vezzi insulsi e frammenti di storia universale. Tendenze estetiche nell'economia del simbolico. Roma, Italia: Luca Sossello Editore.

CONNOR, S. (2014). Effetti personali. Vite curiose di oggetti quotidiani. Milano, Italia: Cortina.

CRISTALLO, V. (2015). La crisi del prodotto nel 'design di prodotto'. Op. cit., (152), 25-37.

DE FUSCO, R. (2008). Parodie del design. Scritti critici e polemici. Torino, Italia: Allemandi.

DORFLES, G. (1972). Introduzione al disegno industriale. Torino, Italia: Einaudi.

ERLHOFF, M. \& MARSHALL, T. (Eds.). (2008). Design Dictionary. Perspectives on Design Terminology, Basel, Switzerland: Birkhäuser.

FONTANILLE, J. (2002). La patina e la connivenza. In E. Landowski \& G. Marrone (a cura di), La società degli oggetti. Problemi di interoggettività (p. 71-95). Roma, Italia: Meltemi.

LEROI-GOURHAN, A. (1977). II gesto e la parola, La memoria e i ritmi (Vol. II). Torino, Italia: Einaudi.

MALDONADO, T. (1976). Disegno industriale: un riesame. Milano, Italia: Feltrinelli.

(1992). Reale e virtuale. Milano, Italia: Feltrinelli.

MARRONE, G. (2002). Dal design all'interoggettività: questioni introduttive. In E. Landowski \& G. Marrone (a cura di), La società degli oggetti. Problemi di interoggettività (p. 9-38). Roma, Italia: Meltemi.

PIZZOCARO, S. (2015). Introduzione agli studi sull'utente. Conoscere gli utenti tra ricerca e design dei prodotti. Milano, Italia: Unicopli.

(a cura di). (2016). Artefatti concreti. Temi di fondamento per il design di prodotto. Milano, Italia: Unicopli.

REDSTRÖM, J. (2008). RE:Definitions of use. Design Studies. 29(4), 410-423. 